\title{
Inflammatory fibroid polyp in the antrum co-occurring with adenomatous polyp in the ascending colon
}

\author{
Jakub Wronecki ${ }^{1}$, Aleksandra Błaszkiewicz ${ }^{2}$, Jarosław Swatek ${ }^{3}$, Barbara Skrzydło-Radomańska ${ }^{1}$ \\ ${ }^{1}$ Department of Gastroenterology and Endoscopy, Medical University of Lublin, Lublin, Poland \\ ${ }^{2}$ Independent Public Hospital No. 4, Lublin, Poland \\ ${ }^{3}$ Department of Clinical Pathomorphology, Medical University of Lublin, Lublin, Poland
}

Gastroenterology Rev 2018; 13 (4): 340-342

DOI: https://doi.org/10.5114/pg.2018.79816

\begin{abstract}
Address for correspondence: Prof. Barbara Skrzydło-Radomańska MD, PhD, Department of Gastroenterology and Endoscopy, Medical University of Lublin, 8 Jaczewskiego St, 20-954 Lublin, Poland, e-mail: gastrol@mp.pl
\end{abstract}

Inflammatory fibroid polyps (IFP), also known as Vanek tumours, are one of the rarest group of gastrointestinal tract polyps. They represent $0.1-3.0 \%$ of all polyps in this organ system [1]. They are defined as benign neoplastic lesions projecting from the submucosa or mucosa, consisting of spindle stromal cells with infiltration of eosinophils. Most common location is the stomach, mainly the antrum (70\%), ileum (19\%), and colon (6\%) [2]. Depending on size and location, IFP can be asymptomatic or occurring with abdominal pain, gastrointestinal bleeding, weight loss, and vomiting. Large polyps of the stomach can cause intermittent obstruction, described as "ball valve syndrome" [3]. There is also the possibility of intussusception presenting with acute abdomen when the polyp arises below the Treitz ligament $[1,4]$.

Our paper presents a case report of a female patient who was diagnosed with IFP co-occurring with adenomatous polyp of ascending colon.

A 59-year-old woman was admitted to the Chair and Department of Gastroenterology with Endoscopic Unit for planned polypectomy. Previously she had been treated in an outpatient clinic, where colonoscopy and esophagogastroduodenoscopy (EGD) were performed three months prior to admission. During colonoscopy no lesions were found up to the splenic flexure, but it was not possible to examine the further part of the colon. During EGD a polyp in the antrum was found and biopsied. Histopathological examination of the specimen revealed chronic gastritis and the features of hyperplastic polyp.

On admission the patient reported a lack of appetite, constipation, and weight loss (16 kg within a year). Physical examination revealed abdominal pain in the upper right quadrant with no abnormal reactions. Esophagogastroduodenoscopy performed in the hospital found a polyp in the antrum of about $10 \mathrm{~mm}$ diameter on a wide base, which was resected using a diathermic snare. Histopathologically, inflammatory fibroid polyp was diagnosed.

Colonoscopy under general anaesthesia was also performed. It revealed a longitudinal polyp, placed on a fold of about $12 \mathrm{~mm}$ in length, which was biopsied. Histopathology report indicated tubular adenoma with high-grade dysplasia. The patient was referred to a surgical clinic for further treatment. Endoscopic mucosal resection was performed resulting in successful removal of the whole lesion, complicated however by profuse bleeding. The patient remains under the care of an outpatient gastroenterology clinic with no recurrence of symptoms after a year of follow-up appointments. No further operations were necessary.

Inflammatory fibroid polyps is a rare polyp of the gastrointestinal tract. The cause of IFP is still unknown [4]. It was first described by Vanek in 1949 as a benign submucosal granuloma consisting of basic fibrous connective tissue with eosinophilic infiltration [5]. Formerly it was known as: Vanek polyp, Vanek tumour, inflammatory pseudotumour, granuloma with eosinophils, eosinophilic granuloma, and polyp with eosinophilic granuloma [6]. In 1953 Helwig and Ranier proposed the name IFP, which is nowadays predominantly used, to highlight the morphology of the polyp $[1,2]$.

The incidence rate is comparable in both men and women, with a slight predominance in men. Inflammatory fibroid polyps are reported in every age group, but the peak of incidence occurs at 50-70 years of age [1]. 
The clinical presentation depends on the size and location of the lesion. It can be asymptomatic or occur with gastrointestinal bleeding, abdominal pain, and vomiting. Polyps located in the upper gastrointestinal (GI) can cause dysphagia and gastroesophageal reflux [7], while those located below the ligament of Treitz can cause obstruction, diarrhoea, and intussusception, which may lead to acute abdomen $[1,4,6]$. Inflammatory fibroid polyps can also imitate acute appendicitis [8, 9]. Asymptomatic polyps are also found incidentally during endoscopy performed for unrelated reasons [8] (Figure 1).

Inflammatory fibroid polyps can be found during endoscopy but can also be found during laparoscopy or laparotomy $[4,9]$. Macroscopically they are usually a single lesions $2-5 \mathrm{~cm}$ in diameter, semipedunculated or sessile, covered with normal mucosa, with possible erosions $[10,11]$. Histologically IFPs are submucosal (but often expand to the mucosa) with a well-developed network of capillaries, characteristic "onion skin" arrangement of spindle cells around vessels, and infiltration of eosinophils and lymphocytes [12]. Histopathologically they should be differentiated with gastrointestinal stromal tumors (GISTs), schwannomas, and inflammatory myofibroblastic tumours [13]. Immunohistochemical staining of IFPs is usually positive for CD-34 and negative for CD-117 and S-100 protein $[12,13]$. This type of polyp is associated with mutation in the PDGFR-A gene, a feature shared with gastrointestinal stromal tumours $[12,13]$. Due to the submucosal character of the lesion, biopsy specimens taken during endoscopy may not confirm the final diagnosis before resection. In such cases endoscopic ultrasonography or computed tomographic imaging can be used [10]. While the most common removal method is endoscopic resection, there have been reports of infiltration of the muscularis propria layer of stomach, which requires a more radical approach and careful examination of surgical margins [13].

Vanek tumours are rare lesions and occur individually, in contrast to the most common singular or multiple (in $40 \%$ of cases) epithelial gastric polyps, known as fundic gland polyps (FGP, Elster glandular cysts) [14, 15]. The genesis of FGPs is not known, and they are treated as hamartomatous lesions or a particular form of hyperplastic polyp [15].

For practical reasons FGPs were divided into sporadic type and those associated with familial adenomatous polyposis (FAP). Sporadic FGPs have no clinical significance but their presence is often the reason for referring patients for endoscopic polypectomy. Because there is no real risk of transforming into stomach cancer in such cases, follow-up endoscopy is not recommended [16]. Studies also did not find increased risk of colorectal adenomas in patients with sporadic FGPS

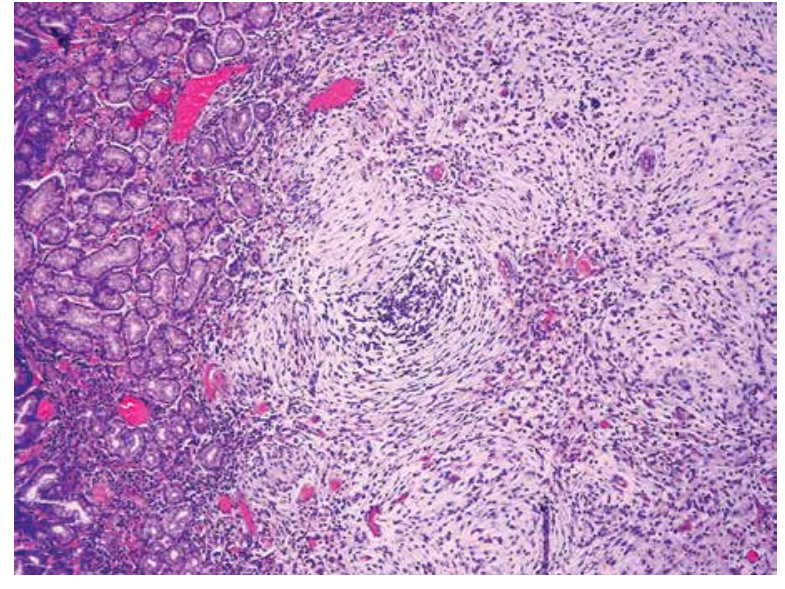

Figure 1. Inflammatory fibroid polyp of the antrum located mostly in the submucosa and expanding to the mucosa. $\mathrm{H}+\mathrm{E}$, magn. 100x

$[16,17]$. In patients with FAP, the incidence rate of FGPS is significantly increased, and they are even treated as a symptom of FAP. Therefore, all patients with incidentally diagnosed FGPs, who are under 40 years old, should undergo colonoscopy $[16,18]$.

Due to the rarity of IFP there is no available literature describing co-occurrence of IFP and other types of polyps; therefore, we can assume that such an occurrence was incidental in the reported case.

\section{Conflict of interest}

The authors declare no conflict of interest.

\section{References}

1. Abboud B. Vanek's tumor of the small bowel in adults. World J Gastroenterol 2015; 21: 4802-8.

2. Helwig EB, Ranier A. Inflammatory fibroid polyps of the stomach. Surg Gynecol Obstet 1953; 96: 335-67.

3. Fleres F, Mazzeo C, leni A, et al. Gastric inflammatory fibroid polyp tumor with acute intestinal obstruction - Vanek's tumor can mimick a giant gastrointestinal stromal tumor or a gastric lymphoma. J Vis Surg 2018; 4: 54.

4. Adams HS, Bergstrom B, Haines B, et al. Inflammatory fibroid polyp: an unusual cause of ileoileal intussusception. Case Rep Surg 2017; 2017: 6315934.

5. Vanek J. Gastric submucosal granuloma with eosinophilic infiltration. Am J Pathol 1949; 25: 397-411.

6. Nonose R, Valenciano JS, Silva CMGD, et al. Ileal intussusception caused by vanek's tumor: a case report. Case Rep Gastroenterol 2011; 5: 110-6.

7. Kwiatkowski AP, Paśnik K. Large inflammatory fibroid polyp of cardia managed laparoscopically - a case report and review of the literature. Videosurgery Miniinv 2014; 9: 623-6.

8. Kordzadeh A. Vanek's tumour mimicking an acute appendicitis. Int J Surg Case Rep 2011; 2: 264-6. 
9. Sánchez-Cifuentes Á, González-Valverde FM, Ruiz-Marín M, et al. Inflammatory fibroid polyp of the appendix or Vanek's tumor. Rev Esp Enferm Dig 2015; 107: 37-8.

10. Fuke H, Hashimoto A, Shimizu A, et al. Computed tomographic image of an inflammatory fibroid polyp of the stomach. Clin Imaging 2003; 27: 400-2.

11. Kröner P, Council L, Mönkemüller K. Endoscopic characterization and resection of Vanek's tumor of the duodenum. Endoscopy 2015; 47 Suppl 1: E408-9.

12. Liu TC, Lin MT, Montgomery EA, et al. Inflammatory fibroid polyps of the gastrointestinal tract. Am J Surg Pathol 2013; 37: 586-92.

13. Harima $\mathrm{H}$, Kimura T, Hamabe $\mathrm{K}$, et al. Invasive inflammatory fibroid polyp of the stomach: a case report and literature review. BMC Gastroenterol 2018; 18: 74.

14. Cao H, Wang B, Zhang Z, et al. Distribution trends of gastric polyps: an endoscopy database analysis of 24121 northern Chinese patients. J Gastroenterol Hepatol 2012; 27: 1175-80.

15. Elster K. Histologic classification of gastric polyps. Curr Top Pathol 1976; 63: 77-93.

16. Genta RM, Schuler CM, Robiou Cl, et al. No association between gastric fundic gland polyps and gastrointestinal neoplasia in a study of over 100,000 patients. Clin Gastroenterol Hepatol 2009; 7: 849-54.

17. Cimmino DG, Mella JM, Luna P, et al. Risk of colorectal polyps in patients with sporadic gastric polyps: a case-control study. World J Gastrointest Endosc 2013; 5: 240-5.

18. Goddard AF, Badreldin R, Pritchard DM, et al. The management of gastric polyps. Gut 2010; 59: 1270-6.

Received: 28.06 .2018

Accepted: 5.10 .2018 